\title{
Recognition and Classification of Various Dance Forms by Using SVM Classifier
}

\author{
Bhavana R.Maale, Pushpanjali
}

\begin{abstract}
Indian traditional dance involves a tough and difficult dance poses and hand gesture and leg movements, so it is a tedious task to recognize and discover human dance movements from video sequences because dance poses are hidden in their heavy makeup and typical costumes and it make nervures for audience who watching dance performance and also to dance fans. In this paper work we have taken into account this problem and made an improvement by using a new classification model. In this new model we demonstrated three varieties of classical dance forms such as Bharathanatium, oddisi, kathak. In Research lab we experimented various form of dance videos and simultaneously carried an investigation on frames (images) generated from uploaded video by using a python integrated shell environment. The new model used a Shannon entropy algorithm for preprocessing of particular dance image and applied a Hu movements for extract a features and this will be issued as input to SVM classifier and then it predict which dance form it is and at same time it display a pixel value of that image with this new model system performance is high recognition accuracy is $94.4 \%$ with SVM classifier.
\end{abstract}

IndexTerms - Frameset Generation, SVM classifier, Python IDLE, Shell environment, Hu movements, Shannon Entropy.

\section{INTRODUCTION}

At present it's getting trend that youths are attracted towards the extra curriculum activities like singing, music and dancing. In dancing is a dramatic art which consists of huge number of human dance, graceful poses and signature steps of dances depends on dance form. The dance is made by different choreographers, dance teacher and dance trainer and every dance is combined with melody song and background noise and setup. Every dance is made in the form of softcopy and recorded by using various video formats like MP4 ,3GP,WEBM,FLY etc. dance video are different from each other by dance songs forms ,formats, music and body part gestures. As the year by year pass everyone attracted towards to videos, YouTube, tube mate and dance clips and reality shows of dances. Now we turned form image/Frame to video dataset generation and for this needed a new technology to show a pure and sharp dance poses from large video. Since

Manuscript revised June 7, 2019 and published on July 10, 2019

Prof Bhavana R.Maale, Department of Studies in Computer Science and engineering, Visvesvaraya Technological University Centre for PG Studies, Kalaburgi, India.

Pushpanjali, Department of Studies in Computer Science and engineering, Visvesvaraya Technological University Centre for PG Studies, Kalaburgi, India.

doi: 10.32622/ijrat.76201967 from 5000 years a dance art is come into existence with traditional songs where as each Indian culture have their own dance songs and poses. Since form many years this problem is taken into an account and various experiment is done on it and each there is improvement in the output result. Now at present paper made a lot of improvement over it by using high end technology.

In this research paper work we focused on the problem of identifying and classifying dance actions form sequence of video of any dance type and dance form. This problem is taken as challenge in present work and done research by using two different expert algorithms whose output is always definite and correct. In the proposed paper is involves about the experiment performed in the lab on short video of Bharathanatium and this tested by using python environment which is easy to use and user friendly as well. In this system as first it is needed to upload video and then simultaneously it generates a thousands of frames (images) and then performed a preprocessing on single selected image by using Shannon entropy and get the output submit to the SVM classifier as input then get predict the dance form with pixel value of image.

In this research paper work we focused on the problem of identifying and classifying dance actions form sequence of video of any dance type and dance form. This problem is taken as challenge in present work and done research by using two different expert algorithms whose output is always definite and correct. In the proposed paper is involves about the experiment performed in the lab on short video of Bharathanatium and this tested by using python environment which is easy to use and user friendly as well. In this system as first it is needed to upload video and then simultaneously it generates a thousands of frames(images) and then performance a preprocessing on single selected image by using a Shannon entropy and get the output submit to the SVM classifier as input then get predict the dance form with pixel value of image.

In [1] SVMC is firstly introduced and verified with set of benchmark dataset of image and it is used in this work and they yield a major furtherance new fusion model and result outcome is based on qualitative and quantity $\mathrm{n}$ datasets. There is major investigation is carried out in [2] about shapes and large pixel size of image which is taken into account by using a algorithm of segmented interactive classifier and in this present proposed work used this technology for accurate and sharp identification images in the sets. The new superior computer vision model [3] is yield with aim to help for e-learning and self learning. This model is suitable for only 
leg movement identification and works with performance accuracy is almost $86.7 \%$ with SVM classifier. More over a Statistical method [4] are taken into an account by principle "object extracting" challenge. The goal of this method is object identification in large video sequence which provides a high mobility. These system utilized daubechies multifarious wavelets transform which helpful for an object pointing in a video. The solution for visual video set[5] introduce with improved presentation that helps for the various applications such as surveillance, monitoring and tracking actions and video clips capturing and editing, storing in lager storage sets.

The major goal of this research is to identify with dance signature steps from dance forms which is in accurate and the purpose system is allows to upload any video formats like WEBM, MP4, 3GP etc. and which is generate frames (images) should in faster and accurate with high image resolution which is built by using new programming language is python. This proposed work will mainly focus on video dataset based on which a high end work is taken place. The scope of our work is helpful for dance beginning learner and dance trainer which they do practice by getting high resolution image sets. The major opportunity of this proposed system is helps for self dance learner and it is utilized to the dance choreographer to make dance with different movements which is mix with music. This system is spreader in vision work in computer science. This System is made with portable python shell environment which coding is easily be done and debugging will takes only a few seconds, so this system is used by anyone anywhere by using a python executable code. The SVM is a good choice and computational complexities are decreases. SVM performs better with clear margin separation in hyper plane and effectively works with multidimensional space.

\section{METHODOLOGY}

The proposed system is involves a library for storage which s used to generated images and uses OpenCV library of python for converting video to dataset of images. The proposed system shows combination of various modules like Upload video, Frame Generation, Preprocessing, Feature extraction and predictions as shown in figure 1.

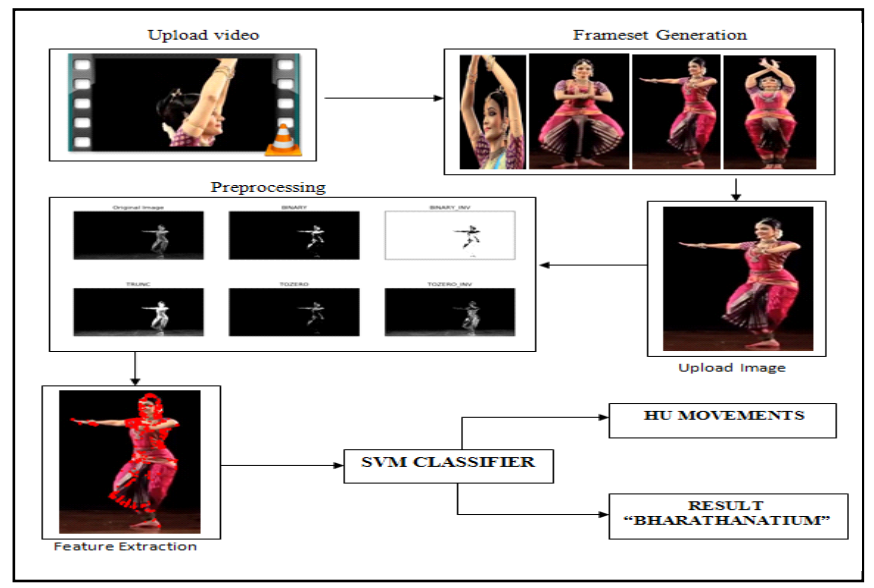

Fig.1. Proposed System Architecture

\section{A. Video to Frameset Generation}

To Generate a Framesets of uploaded video of any format can be done by using python OpenCV library function. Each generated frame/image is stored in database of "Frames" with label from frame0 to Frames. Each frame has their own key properties like pix el value, size, and image with different styles. These images are helps for further research work.

\section{B. Preprocessing}

The preprocessing is a process of improvement of images and notifies distortion to enhance some frame features and for preprocessing "Shannon Entropy" algorithm is used in this paper. The Preprocessing module which is Convert from original image as shown in figure 2 into gray color image as show in figure 3 .

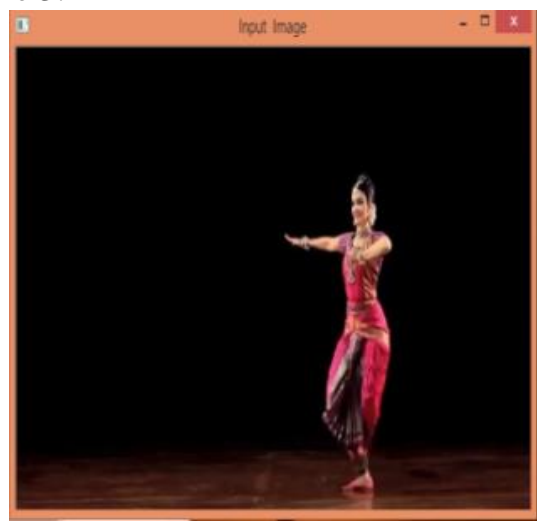

Fig.2. Original Image

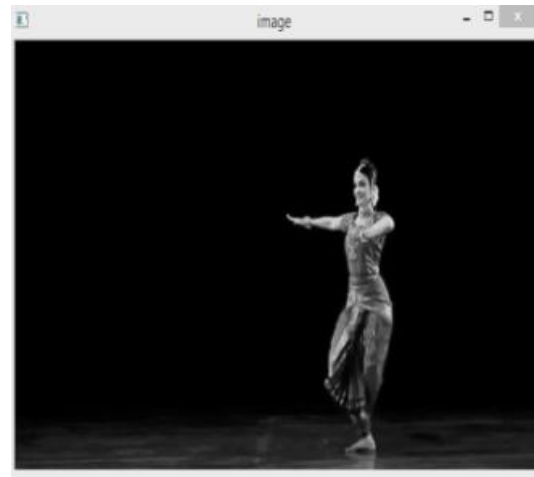

Fig.3. Gray Color Image

The Shannon's entropy is helps to extract various feature by calculate a training data and input the testing data. This algorithm is yield an accurate training data to calculate a dance forms.

Shannon's Entropy Equation:

$$
H(X)=-\sum_{i=0}^{N-1} b i \quad \log _{2} b i
$$

In this equation, probability for a specified symbol is pi. To perform $\log _{2}$ with other base value of $\log$ such as 10 or e such as:

$\log _{2}(n)=\frac{\log _{a}(n)}{\log _{a}(2)}$ 
The least average number of bits per symbol is:

NumBits $=[H(X)]$

The preprocessing helps to describes various thresholding operations as binary, binary-inverse, Truncate, To-Zero and To-Zero-Inverse. The binary threshold is first thresholding operation of preprocessing which coverts original image binary value two values are 0 's and 1's means white and binary value 0's means black. This Binary_Inv thresholding operation which is total opposite to Binary operation and it treats 0 's means white and binary value 1 's means black. This truncate thresholding operation compares pixel value less than threshold value that is 127 than it treats as 0 's(black) and greater than threshold value it is 1 's(white). ).TOZERO is thresholding operation compares if image pixel less than value of threshold it is full black that its value is 0 and if greater than pixel than threshold value it is full black. The TOZERO_INV is thresholding operation compares if image pixel greater than threshold value it is full black that its value is 0 and if less than pixel than threshold value it is full black and its value is 1 as depicted in figure 4 .
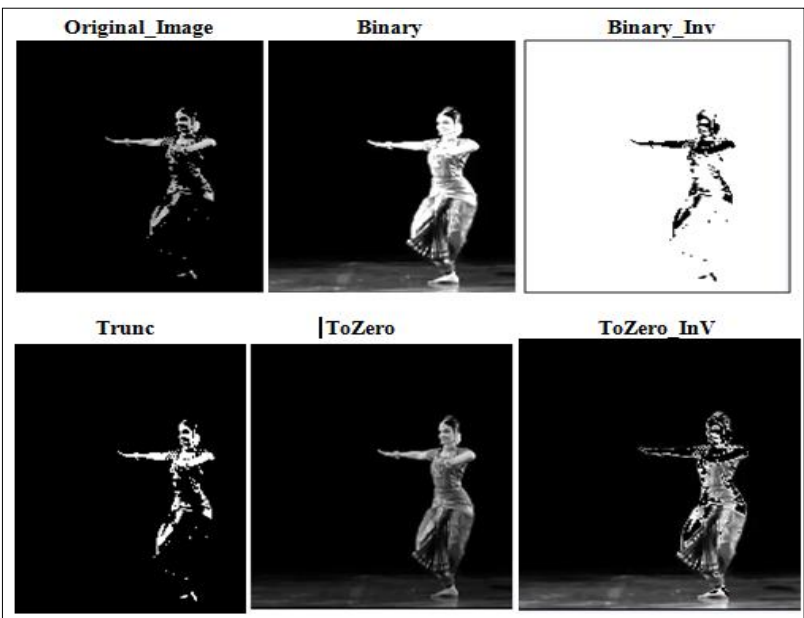

Fig.4. Thresholding Operations

\section{Feature Extraction}

This Feature extraction module is most important module in proposed system. This module is also called as "feature selection phase". This module extract features like hand and leg movements and face gesture and movement of any body part are recognized using $\mathrm{Hu}$ movements as shown in figure 5 . The Hu movements are mainly used to get the accurate shape of the work. In system a feature extraction involve with a technique called as "SVM (Support Vector Machine)".The SVM is discriminative classifier which normally defined by a separable hyper lane. A new example can be defined by given training/testing data to this algorithm that highlighted on optimal hyper plane surface. To divide two classes by a hyper-line we are using a proposed system classifier.

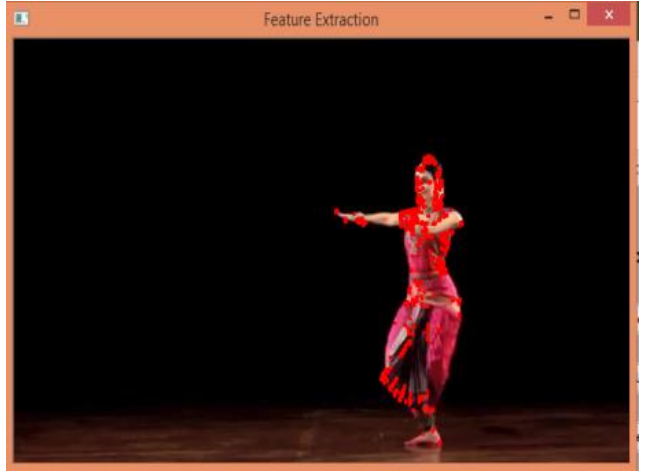

Fig.5. Feature Extracted Image

\section{Predict}

It is last module of proposed system which predict which kind of dance it is whether it is Bharathanatium ,kathak, oddisi as shown in figure 6 and it predict the frame based on the pixel value of image as shown in figure 6 . If the uploaded image pixel value is more than or equal to 8 than it "BHARATHANATIUM" as it predict dance and pixel value is more than or equal to 11 than it predict as "KATHAK" dance, and If value is exceeds than or equal to 14 than it predict as "ODISSI" dance, finally If the no image uploaded the than it predict as "NO PREDICT/CLASSIFIED" dance.

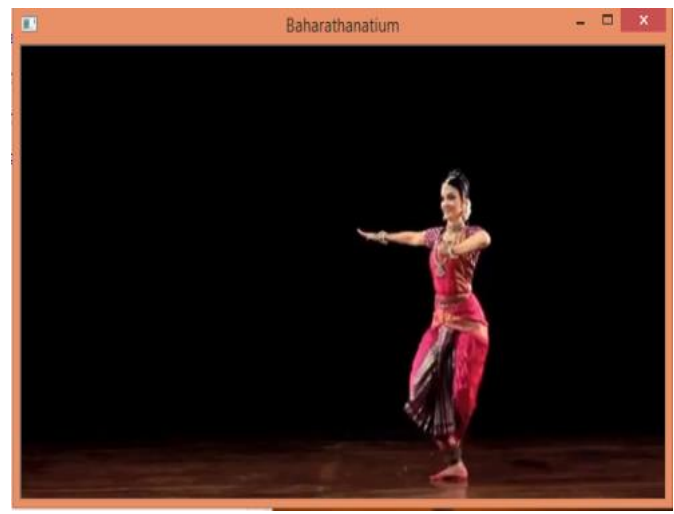

Fig.6. Predict Dance form as Bharathanatium

\section{RESUL AND ANALYSIS}

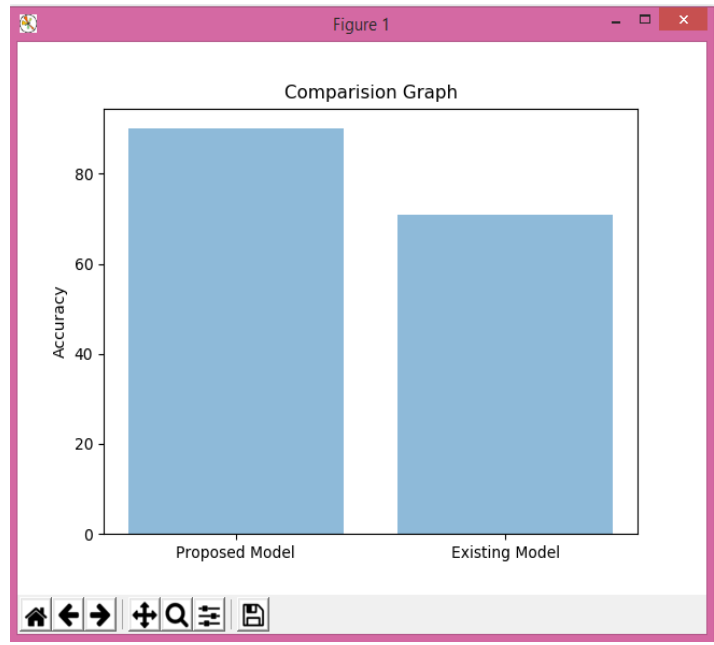

Fig.7. Comparison result Graph 
The result graph is show the accuracy of the proposed system and it verify with the existing system accuracy as shown in above graph which compared with existing system accuracy is up to $74.8 \%$. The proposed system is accuracy and recognizing as well as identifying the different dance forms poses and using Shannon entropy algorithm performance for high recognition accuracy $94.4 \%$ with SVM classifier and each of accuracy value is increased with this classifier with help of comparison result graph as shown in figure 7.The future work of proposed systems is made by combined with hardware and software requirements in considers and performed a research on various traditional Indian dance classifications with SVM. In future it is possible to work with online or live dance video by using different and latest classifier. In future it is need to predict with high accuracy and latest dance like Indian as well as western dance as well and any dance forms in the world.

\section{CONCLUSION}

The proposed systems have various applications dance training and choreography of songs and this system helps to get the accurate signature of dance poses of dance. In this proposed system performed a work on three different Indian dances. This system used a Hu movement which is used to get the correct signature poses and by using SVM which helps to notify and identify the dance actions and this system setup is based python environment which is user friendly. The proposed system performed measures up to $94.4 \%$ with help of SVM classifier.

\section{REFERENCES}

[1] Chirag I Patel, Sanjay Garg, Tanish Zaveri, Asim Banerjee, and Ripal Patel, "Human action recognition using fusion of features for unconstrained video sequences," Computers \& Electrical Engineering, 2016.

[2] Kumar, K. V. V., P. V. V. Kishore, and D.Anil Kumar, "Indian Classical Dance Classification with Adaboost Multiclass Classifier on Multifeature Fusion," Mathematical Problems in Engineering ,2017.

[3] Sriparna Saha, Shreya Ghosh, Amit Konar, "A Study On Leg Posture Recognition From Indian Classical Dance Using Kinect Sensor,” 2018.

[4] Ming-Kuei Hu, "Visual pattern recognition by moment invariants," IRE transactions on information theory, 8(2):179-187, 196.

[5] Manish Khare, Rajneesh Kumar Srivastava, and Ashish Khare, "Object tracking using combination of daubechies complex wavelet transform and Zernike moment. Multimedia Tools and Applications,' 76(1):1247-1290, 2017.

\section{AUTHORS PROFILE}

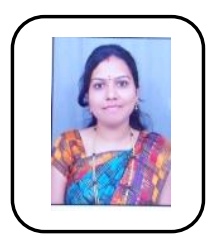

Mrs. Bhavana R.Maale has completed B.E and M.TECH from PDA College of engineering Kalaburagi and pursuing $\mathrm{Ph} . \mathrm{D}$ at research centre PDA college of Engineering Kalaburagi. Presently working as a assistant professor in the department of computer science \& Engg Kalaburagi Visvesvaraya Technological University, Postgraduate Centre, Kalaburagi, India.

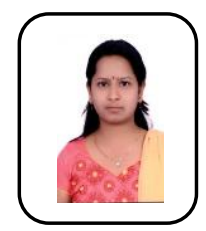

Miss Pushpanjali has completed B.E(CSE) from PDA College of engineering Kalaburgi and pursuing M.TECH in computer science and Engineering at Visvesvaraya Technological University, Postgraduate Centre, Kalaburgi, India. She is having professional membership of ISTE. 